\title{
DRUG NANONIZATION: AN OVERVIEW OF INDUSTRIALLY FEASIBLE TOP-DOWN TECHNOLOGIES FOR NANOCRYSTAL PRODUCTION
}

\author{
Sunita Dahiya* \\ Department of Pharmaceutical Sciences, School of Pharmacy, University of Puerto Rico, Medical Sciences \\ Campus, San Juan, PR-00936, United States \\ *E-mail: sunita.dahiya@upr.edu \\ Tel.: +17872456385
}

Received: May 18, 2017 / Revised: Jul 16, 2017 / Accepted: Jul 18, 2017

Many drugs are associated with one or more problems in their delivery including, poor bioavailability, in vivo stability, solubility, intestinal absorption, sustained and targeted delivery to site of action, therapeutic effectiveness, side effects and plasma fluctuations of drugs which either fall below the minimum effective concentrations or exceed the safe therapeutic concentrations. Extensive research efforts have been reported including micronization, salt formation, $\mathrm{pH}$ modification, solid dispersions, complexation, solubilization, hydrotropy etc for addressing one or more of these challenges in order to achieve desired formulation performance. Many of these techniques have been commercialized and still under continuous research. In spite of their potential for improved drug delivery, they suffer from one or more limitations and could not be exploited fully. Recently, various nanonization strategies have emerged to increase the dissolution rates and bioavailability of numerous drugs that are poorly soluble in water. These technologies have been proved to be highly effective not only for improving poor aqueous solubility and bioavailability problems but also in dealing with other formulation challenges. This article overviews the potential of nanonization to overcome many problems associated with modern drug delivery and addresses the salient features of the industrially feasible top-down technologies involved in production of nanocrystals.

Key words: Nanocrystal, Nanosizing, Top-down techniques, Nanonization, Nanoparticles.

\section{INTRODUCTION}

Poorly-water soluble compounds comprise a significant and growing percentage of the industries' drug development pipeline and are viewed as highly challenging development candidates. To address this issue, formulation strategies for molecules which belong to BCS class II (poorly soluble and permeable) and Class IV (poorly soluble and impermeable), have been of greatest interest to the formulation scientists. The use of particle size reduction approaches to form stable nanometer size drug nanosuspensions or nanoparticles is a relatively newer formulation strategy (Dahiya et al 2007). A number of methodologies can be adapted to improve solubility of poor water soluble drug and further to improve its bioavailability. The techniques generally employed for solubilization of drug includes micronization, salt formation, chemical modification, $\mathrm{pH}$ adjustment, solid dispersion, complexation, co-solvency, micellar solubilization, hydrotropy, self-emulsifying drug delivery, microemulsions etc. In past, various research efforts have been reported with potential results employing solid dispersion and 
cyclodextrin technology (Dahiya, 2017; 2010; Radhika et al 2015; Patel et al 2015; Dahiya and Onker, 2015; Dahiya et al 2015; Prusty, 2014; Dahiya and Tayde, 2013; Pabreja and Dua , 2011; Dahiya and Kaushik, 2010; Pathak et al 2008; Dahiya et al 2008; Dahiya and Pathak, 2007, $2006 \mathrm{a} ; 2006 \mathrm{~b})$. In spite of potential results achieved, these techniques are associated with one or more limitations which led to their limited commercialization.

Some of the challenges of most drug delivery systems include problems such as poor bioavailability, in vivo stability, solubility, intestinal absorption, sustained and targeted delivery to site of action, therapeutic effectiveness, side effects and plasma fluctuations of drugs which either fall below the minimum effective concentrations or exceed the safe therapeutic concentrations (Khanbabaie and Jahanshahi, 2012). Nanotechnology in drug delivery is an approach designed to overcome these problems due to the development and fabrication of nanostructures at submicron scale and nanoscale which are mainly polymeric and have multiple advantages. Nanostructures have the ability to protect drugs encapsulated within them from hydrolytic and enzymatic degradation in the gastrointestinal tract; target the delivery of a wide range of drugs to various areas of the body for sustained release and thus are able to deliver drugs, proteins and genes through the peroral route of administration. They deliver drugs that are highly water insoluble; can bypass the liver, thereby preventing the first pass metabolism of the incorporated drug. They increase oral bioavailability of drugs due to their specialized uptake mechanisms such as absorptive endocytosis and are able to remain in the blood circulation for a longer time, releasing the incorporated drug in a sustained and continuous manner leading to less plasma fluctuations thereby minimizing side-effects caused by drugs. Due to the size of nanostructures, they are able to penetrate into tissues and are taken up by cells, allowing efficient delivery of drugs to sites of action. The uptake of nanostructures was found to be 15-250 times greater than that of microparticles in the 1-10 $\mu \mathrm{m}$ range. Literature has reports indicating that delivered drugs that are highly water insoluble; can bypass the liver, thereby preventing the first pass metabolism of the incorporated drug (Ochekpe et al 2009).

At the beginning of the 1990s, the drug nanocrystals were developed as more efficient approach to increase drug solubility and dissolution velocity. Instead of micronizing the drug powder, it is nanonized leading to nanocrystals with a typical size of about $200 \mathrm{~nm}$ up to approximately $600 \mathrm{~nm}$. The success of this approach is clearly documented by the short time between invention and the first product on the market in the year 2000 (Rapamune $\AA$, Wyeth). With continuation, many products are on the market as well as in various clinical phases. Until now, abundant work has been done in developing production technologies for the nanocrystals. The state of the art first generation technologies such as bottom up (precipitation) and top down (comminution), to the second, improved and smarter drug nanocrystal generation known as second generation technology (SmartCrystals). The review focuses on nanonization concept with special emphasis on industrially feasible top-down Nanocrystals production technologies.

\section{Concept of nano, nanosizing and nanonization}

Nanonization is a term for reducing particle size to the nanometer range and brings about several advantages. It further increases the surface area of active pharmaceutical ingredient (API) which could result in enhanced solubility and drug bioavailability. Reducing the particle size of an API possessing poor solubility characteristics can lead to higher specific surface area, thereby increasing bioavailability and dissolution rate. Due to the increased bioavailability a lower amount of API is required which in turn leads to a more cost-efficient product with less risks and side effects for the patient. The term 'nanosizing' is used to reference particle sizes in the submicron range, typically 100-200 nm (nanometers, or $1 / 1000^{\text {th }}$ of a micrometer) in size. Nanosizing is typically accomplished by milling the compound in a stabilized solution to the targeted size, which can be further processed into the final dosage form. The process of converting particles to nanosize is known as nanonization. Recently, various nanonization strategies have emerged to increase the dissolution rates and bioavailability of numerous drugs that are poorly soluble in water. These strategies include increasing the surface area to volume ratios of drug powders, changing the crystalline forms and designing novel nanomaterials that can act as carriers for controlled release (Junghanns and Muller, 2008). Nanonization can result in improved drug 
solubility and pharmacokinetics, and it might also decrease systemic side-effects (Riehemann et al 2009). Nanonization of hydrophobic drugs generally involves the production of drug nanocrystals through either chemical precipitation or disintegration (Junghanns and Muller, 2008). Alternatively, nanotechnologybased drug delivery systems such as nanoemulsions and polymeric micelles can be used. During the past decade, several drug nanoformulations have been clinically approved or are under clinical investigation (Junghanns and Muller, 2008).

\section{Distinct features of nanosized drugs}

Drug nanoparticles are a formulation principle for all poorly soluble drugs for which the dissolution velocity is the rate limiting step for absorption and thus the reason for a too low oral bioavailability. This means nanocrystals are made essentially from poorly soluble drugs; water soluble drugs cannot be formulated as nanocrystals at least in aqueous dispersion medium. The increase in surface area leads to an increase in the dissolution velocity according the Noyes-Whitney equation. A fact in the past often overlooked is the increase in the saturation solubility of nanonized compounds compared to micrometer particles, precisely the kinetic saturation solubility increases. The basis for this is the Kelvin equation describing the vapour pressure as a function of the curvature of liquid droplets in a gas phase. Compared to micrometer crystals the nanocrystals lead to a supersaturated solution. This situation is metastable, that means as a function of time crystallisation will be initiated, large crystals will precipitate and the system returns to the thermodynamically stable state of the saturation solubility of micrometer crystals. However, in general duration of this supersaturated state is sufficient for oral absorption. The excellent advantages offered by nanonized drug is possible due to its distinct features i.e. increased saturation solubility, increased dissolution velocity and increased adhesiveness to surfaces/cell membranes.

\section{Nanocrystalline particles vs NanoCrystal}

Drug nanocrystals are nanoparticles with a crystalline character. The size of a particle to be classified as a nanoparticle, depending on the discipline, e.g. in colloid chemistry particles are only considered as nanoparticles when they are in size below $100 \mathrm{~nm}$ or even below $20 \mathrm{~nm}$.
Based on the size unit, in the pharmaceutical area nanoparticles should be defined as having a size between a few nanometers and $1000 \mathrm{~nm} \mathrm{(=}$ $1 \mu \mathrm{m})$. Drug nanocrystals are particles made from $100 \%$ drug; typically they are stabilized by surfactants or polymeric steric stabilizers. Drug nanocrystals do not possess any carrier material as in matrix nanoparticles consisting e.g. of a polymeric matrix (polymeric nanoparticles), or a lipidic matrix (nanoemulsions, liposomes and lipid nanoparticles). The nanocrystals are typically produced in a liquid dispersion medium, i.e. the nanocrystals are suspended in the liquid. Dispersion of drug nanocrystals in liquid media leads to so called "nanosuspensions" in contrast to the "microsuspensions" or "macrosuspensions". The dispersed particles need to be stabilized, such as by surfactants or polymeric stabilizers. Dispersion media can be water, aqueous solutions or nonaqueous media (e.g. liquid polyethylene glycol (PEG), oils). Depending on the production technology, processing of drug microcrystals to drug nanoparticles can lead to an either crystalline or to an amorphous product, especially when applying precipitation. NanoCrystal is a patent protected technology (Liversidge et al 1992) in which the drug nanoparticles are obtained by subjecting the drug to media milling (e.g. water, stabilizer solution or buffer). High energy and shear forces generated as a result of impaction of the milling media or beads with the drug providing necessary energy input to disintegrate the microparticle drug into nanonized particles. This technique provides advantage of producing very viscous suspensions with $20-30 \%$ higher solid content that cannot be produced with HPH, which can be further diluted with stabilizer solution to produce desired concentration of nanosuspension. The major concern with this method is the residues of milling media remaining in the finished product could be problematic for administration. This technology was extended further by Muller group where nanosuspension prepared by combination technology (CT) process, milling followed by $\mathrm{HPH}$, to get more stable nanosuspension.

The pharmaceutical benefits of nanocrystals include improvement in formulation performance, such as enhanced dissolution velocity and saturation solubility, reproducibility of oral absorption, improved dosebioavailability, proportionality and increased patient compliance via reduction of number of 
oral units to be taken. Nanocrystal serves as ideal delivery system for oral drugs having the dissolution velocity as rate limiting step for absorption, i.e. drugs of the biopharmaceutical classification system (BCS) class II and IV. In addition, nanocrystals can be injected intravenously as aqueous nanosuspensions. The first product Emend ${ }^{\circledR}$ was on the market in 2000. Nanocrystals, a new carrier-free colloidal drug delivery system with a particle size ranging from 100 to $1000 \mathrm{~nm}$, is thought as a viable drug delivery strategy to develop the poorly soluble drugs, because of their simplicity in preparation and general applicability.

Nanocrystals fundamentally possess two most important properties; increase in dissolution velocity and increase in saturation solubility, which are considered to be the main reasons for the increased dissolution velocity and thus increased bioavailability.

\section{Nanocrystal production technologies}

There are several production techniques to produce drug nanocrystals. Two basic approaches are involved in production of nanocrystals,

○ the top-down technologies (comminution)

$\circ$ the bottom-up technologies (controlled precipitation/crystallization) and

○ combination technologies

The industrially relevant methods are the top down technologies starting from a large-size drug powder to be reduced in size. The bottom up technologies (starting from a dissolved molecule, precipitation) include the need for solvent removal, the difficulty in controlling the process, and the fact that many poorly soluble drugs are poorly soluble not only in aqueous, but also organic media. The drug is dissolved in a solvent and subsequently added to a nonsolvent, leading to the precipitation of finely dispersed drug nanocrystals. Typically, the drug nanocrystals are generated in a liquid dispersion medium (e.g. by precipitation or a disintegration process). The obtained product from this process is a suspension of drug nanocrystals in a liquid stabilized by a surfactant or polymer (socalled 'nanosuspension'). The drug nanocrystals can be administered using very different administration routes. Oral administration is possible as a suspension. More patient convenient dosage forms can be produced by transferring the liquid nanosuspensions to solid dosage forms, i.e. tablets or pellets or granulate containing capsules. In addition, because of their small size, the nanosuspensions can be injected parenterally, especially intravenously. The schematic of basic nanocrystal production technology is depicted in Figure 1 (Srivalli and Mishra, 2016).
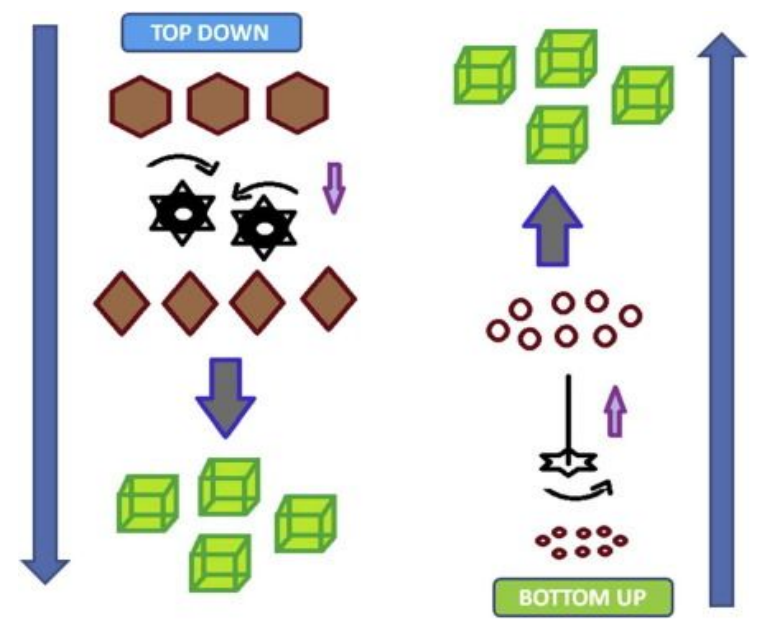

Figure 1. Basic approaches for nanocrystal production technology (NPT)

\section{First generation top down technologies for nanocrystal production}

In the top down technologies, one starts from large crystals in the micron range and goes down to the nanodimension by diminuting the crystals, i.e. performing a milling process. Many techniques including dry milling, wet milling, media milling (pearl/bead milling), high pressure homogenization, and cryogenic milling have been attempted and investigated for various drugs. Among these, two major processes extensively used are pearl milling and high pressure homogenization. All the aforementioned technologies allow nanocrystals production in the size range of about $200 \mathrm{~nm}$ to $1000 \mathrm{~nm}$. When moving toward or below 100 $\mathrm{nm}$, the processes become more tedious. Even running up to 20 passages changes very little in the size of the bulk population after about 10 passages. With small milling beads of $50 \mu \mathrm{m}$, there is a lack of large-scale production lines using such small beads, thereby developing and building them would be relatively costly. This means that larger quantities of ultra-fine drug nanoparticles cannot be produced with reasonable efforts. High pressure homogenization (HPH) is limited by the pressure applicable in production lines under industrial conditions. For many drugs, it was observed that the smallest achievable size was in the range 150-200 nm when applying high pressure 
homogenization. The 'standard' production conditions that are often applied are 1500 bar and 20 homogenization cycles, sometimes even 40 homogenization cycles.

Increasing the homogenization pressure from 1500 to 3000 bar and above had very little effect on further reducing the size. Therefore, there is a need for process modifications available making ultra-fine drug nanoparticles more accessible. A brief overview of first generation top-down techniques is summarized in Table 1.

Table 1. Summary of top down techniques

\begin{tabular}{|c|c|}
\hline Technique & Remarks \\
\hline Dry milling (e.g. jet milling) & $\begin{array}{l}\text { Nanosuspensions in this case are prepared by dry } \\
\text { grinding of poorly soluble drugs with soluble } \\
\text { polymers and copolymers. Polymers and co-polymers } \\
\text { like polyvinylpyrrolidone (PVP), sodium } \\
\text { dodecylsulfate (SDS), polyethylene glycol (PEG), } \\
\text { hydroxypropyl methylcellulose (HPMC), and } \\
\text { cyclodextrin derivatives are used in dry co-grinding } \\
\text { techniques for preparation of nanosuspensions. }\end{array}$ \\
\hline Wet milling & $\begin{array}{l}\text { Wet milling involves size reduction of drug particles } \\
\text { suspended in a liquid medium that may be aqueous or } \\
\text { non-aqueous in nature. Wet milling is particularly } \\
\text { suited for potent drugs and drugs which possess high } \\
\text { residual moisture contents ( }>50 \% \text { moisture) because } \\
\text { dry milling may be problematic for drugs of this } \\
\text { nature Drug particles are dispersed in } \\
\text { surfactant/stabilizer solution to obtain } \\
\text { macrosuspension which is further subjected to milling } \\
\text { energy. }\end{array}$ \\
\hline Media milling & $\begin{array}{l}\text { Media milling can be considered a modernized version } \\
\text { of the ball mill. In media milling, mechanical attrition } \\
\text { and impaction of the suspended drug particles are } \\
\text { brought about by grinding balls, often termed as the } \\
\text { milling media, constructed out of a variety of material } \\
\text { such as glass (yttrium-stabilized), zirconium oxide, } \\
\text { ceramics or highly cross-linked polystyrene } \\
\text { resins [ }\end{array}$ \\
\hline Pearl mill/bead mill & $\begin{array}{l}\text { In media milling, when Pearl balls and beads are used, } \\
\text { the techniques are termed pearl and bead milling } \\
\text { respectively. A low energy milling process using } \\
\text { milling pearls of } 0.2 \mathrm{~mm} \text { to } 0.6 \mathrm{~mm} \text { size moved by } \\
\text { agitator. The crystals are ground between the moving } \\
\text { pearls and the resulting product is nanosuspensions. } \\
\text { Commercially, this is the most successful technique }\end{array}$ \\
\hline High pressure homogenization & $\begin{array}{l}\text { The drug powder is first dispersed into an aqueous } \\
\text { surfactant solution and passed through a homogenizer } \\
\text { to obtain a desired size range. The process uses a jet } \\
\text { stream homogenizer that is Microfluidizer. The high } \\
\text { energy fluid stream of suspension collides; in the } \\
\text { collision zone, the crystals are diminuted by collision } \\
\text { and cavitation. The pressures, number of cycles, and } \\
\text { concentration of drug are the factors that dictate the } \\
\text { final product. The advantages include homogenous } \\
\text { particle size distribution, reproducibility, lower } \\
\text { production time, and continuous production. } \\
\text { Technology is called IDD technology. }\end{array}$ \\
\hline
\end{tabular}




\begin{tabular}{|c|c|}
\hline Piston-gap homogenizer & $\begin{array}{l}\text { An alternative to high energy method. The suspension } \\
\text { passes with a high velocity (e.g. } 500 \mathrm{~m} / \mathrm{s} \text { ) a small gap, } \\
\text { e.g. } 10 \text { microns in height. In the gap, the crystals are } \\
\text { diminuted by cavitation, collision of crystals with each } \\
\text { other, the steel wall and the shear forces of the liquid. } \\
\text { Trade name of this technology is DissoCubes }{ }^{\circledR} \text {. } \\
\text { Typical production conditions are } 1500 \text { bar and up to } \\
20 \text { passes through the high pressure homogenizer. All } \\
\text { homogenization processes are performed with water } \\
\text { suspension. }\end{array}$ \\
\hline Cryogenic milling & $\begin{array}{l}\text { Size reduction begins when the externally-applied } \\
\text { stress induces sufficient strain within the particles and } \\
\text { causes the formation of cracks. The cracks are then } \\
\text { propagated through lines of weaknesses in the } \\
\text { material, with new cracks being initiated and } \\
\text { perpetuated along the way at other discontinuities. } \\
\text { Cryogenic milling or cryomilling in short, is a size } \\
\text { reduction method specially catered to soft, } \\
\text { elastic/plastic, nonbrittle and thermolabile materials. } \\
\text { Cryomilling enables the production of both micron } \\
\text { and nano-sized particles but it has not been widely } \\
\text { adopted in the pharmaceutical industry for the milling } \\
\text { of drugs. }\end{array}$ \\
\hline
\end{tabular}

The known limitations of the first generation/ standard standard processes (WBM, HPH) for the production of drug nanocrystals are the necessity of a micronized drug as the starting material and the long runtimes for the top-down equipment. The combinative particle size reduction techniques have been developed to overcome these drawbacks and to improve the particle size reduction effectiveness of the standard processes.

\section{Second generation/combinative nanocrystal production technologies}

Second generation technologies employ combination technologies and have been developed to obtain nanocrystals with improved properties. The SmartCrystal approach or technology is a toolbox of different combination processes for the production of nanocrystals. Within SmartCrystal toolbox, process variations can be chosen e.g. homogenization in waterethanol mixtures or addition of additives which promote crystal dilution (Keck et al 2008). Nanocrystals of the second generation can be produced using modifications of the basic production technologies. These are, in general, combination technologies. Nowadays, five combinative methods are known: NANOEDGE (microprecipitation followed by a high-energy step such as HPH), H69 (microprecipitation immediately followed by HPH, also called "cavi- precipitation"), H42 (spray-drying followed by $\mathrm{HPH}$ ), H96 (freeze-drying followed by HPH), and the CT combinative technology (media milling followed by HPH) (Shegokar and Muller, 2010). The approach of the SmartCrystal process is to modify the starting material in such a way that it can be better broken down by high pressure homogenization. This means that the SmartCrystal technology is basically a combination technology, a first pre-treatment step is combined with a second high pressure homogenization step (Table 2). The improvements achieved through these technologies mainly include faster production of nanocrystal, higher physical stability, improved in vivo performance of smartcrystals. Combinative processes such as the H42, H96, H69, and the NANOEDGE technologies enable the direct processing of a drug solution after synthesis without previously performing a crystallization step. However, as the H69 and NANOEDGE technologies involve the precipitation of particles in liquid media that usually contain organic solvents, these nanosuspensions are not ready to be used further. Extra drying steps need to be performed to eliminate the organic solvent content, which makes the process longer, more expensive, and more complicated regarding regulatory aspects (Shegokar and Muller, 2010; Teagarden and Baker, 2002). 
Table 2. Some SmartCrystal processes

\begin{tabular}{|c|c|c|}
\hline Process & Pre-treatment & Main treatment \\
\hline H42 & Spray-drying & High pressure homogenization \\
\hline H69 & Precipitation & High pressure homogenization \\
\hline H96 & Lyophilization & High pressure homogenization \\
\hline Combination Technology (CT) & Bead milling & High pressure homogenization \\
\hline NANOEDGE & Microprecipitation & High pressure homogenization \\
\hline
\end{tabular}

On the contrary, when employing the H42 and H96 technologies, the organic solvent necessary to dissolve the poorly soluble drugs is eliminated during the bottom-up step. In this manner, the nanosuspensions produced with the dried intermediates can be directly used or downstreamed for the production of solid dosage forms (Amidon et al 1995; Keck, 2009). In general, the combinative particle size reduction processes perform faster than the standard methods to produce nanosuspensions and achieve smaller final mean particle sizes.

\section{CONCLUSION}

Drug nanocrystals are considered as one of the most important formulation approaches for poorly soluble drugs when simple approaches such as solubilization, self-emulsifying drug delivery systems (SEDDS) and micro-emulsions, do not work. As the optimized nanocrystals formulations, this approach can reduce distinctly

\section{REFERENCES}

Amidon GL, Lennernas H, Shah VP, Crison JR. Theoretical basis for a biopharmaceutic drug classification: The correlation of in vitro drug product dissolution and in vivo bioavailability. Pharm. Res. 1995;12(3):413-20. [DOI: 10.1023/A:1016212804288]

Dahiya S. Fundamentals of Solubility Enhancement Techniques: What do We Need to Consider? Bull. Pharm. Res. 2017;7(1):138. [DOI: 10.21276/bpr.2017.7.1.1]

Dahiya S. Studies on formulation development of a poorly water-soluble drug through solid dispersion technique. Thai J. Pharm. Sci. 2010;34(2):77-87.

Dahiya S, Kaushik A. Effect of water soluble carriers on dissolution enhancement of aceclofenac. Asian J. Pharm. 2010;4(1):34-40. [DOI: 10.4103/0973-8398.63984]

Dahiya S, Kaushik A, Pathak K. Improved pharmacokinetics of Aceclofenac immediate release tablets incorporating its inclusion complex with HP- $\beta$-CD. Sci. Pharm. 2015;83(3):501-10. [DOI: 10.3797/scipharm.1509-07]

Dahiya S, Onker R. Influence of polymethacrylates and compritol on release profile of a highly water soluble drug - Metformin hydrochloride. Ars. Pharm. 2015;56(1):27-31. [DOI: 10.4321/S2340-98942015000100004]

Dahiya S, Pathak K. Influence of amorphous cyclodextrin derivatives on aceclofenac release from directly compressible tablets. Pharmazie 2007;62(4):278-83. [DOI:10.1691/ph.2007.4.6129]

Dahiya S, Pathak K. Physicochemical characterization and dissolution enhancement of aceclofenac-hydroxypropyl $\beta$ cyclodextrin binary systems. PDA J. Pharm. Sci. Tech. the side effects, and therefore, they might also replace existing products. First generation standard top down technologies produce nanocrystals in the size range of 200-1000 nm. These processes become more tedious when moving towards and going below $100 \mathrm{~nm}$. However, ultra-fine drug nanocrystals with a size below $100 \mathrm{~nm}$ are clearly superior to the larger nanocrystals of the first generation and offer many potential advantages. In this context, second generation combinative technologies such as H42, H69 and H96 production, processes have been developed to provide ultra-fine nanocrystals on the lab scale. However, these processes still need to be established on a larger scale. Although the costs for these technologies is a considerable parameter; if these technologies enable highly promising new chemical entity to be put as product on market covering a real therapeutic need, the cost factor could be neglected.

2006;60(6):378-88.

Dahiya S, Pathak K. Dissolution enhancement of aceclofenac by $\beta$-cyclodextrin complexation. J. Pharm. Res. 2006;5(4): 99-103.

Dahiya S, Pathak K, Dahiya R, Pathak D. Nanoparticle engineering processes: Industrial technologies for delivery of poorly water soluble drugs. Egypt. Pharm. J. (NRC) 2007;6(2):87-101.

Dahiya S, Pathak K, Sharma R. Development of extended release coevaporates and coprecipitates of promethazine $\mathrm{HCl}$ with acrylic polymers: Formulation considerations. Chem. Pharm. Bull. (Tokyo) 2008;56(4):504-8. [DOI:10.1 248/cpb.56.504]

Dahiya S, Tayde P. Binary and Ternary Solid Systems of Carvedilol with 2-Hydroxypropyl- $\beta$-cyclodextrin and PVP K30. Bull. Pharm. Res. 2013;3(3):128-34.

Junghanns JAH, Muller RH. Nanocrystal technology, drug delivery and clinical applications. Int. J. Nanomedicine 2008;3(3):295-309.

Keck C, Kobierski S, Mauludin R, Muller RH. Second generation of drug nanocrystals for delivery of poorly soluble drugs: SmartCrystal Technology. Dosis 2008; 24(02):124-8.

Keck CM. Nanocrystals as Novel Approach for Dermal Delivery, Annual Meeting of the German Pharmaceutical Society (DPhG), Jena/Germany, 2009.

Khanbabaie R, Jahanshahi M. Revolutionary impact of nanodrug delivery on neuroscience. Curr. Neuropharmacol. 2012;10(4):370-92. [DOI: 10.2174/157 


\section{3]}

Liversidge GG, Cundy KC, Bishop JF, Czekai DA. Surface modified drug nanoparticles. US Patent 5, 145, 684, 1992.

Ochekpe NA, Olorunfemi PO, Ngwuluka NC. Nanotechnology and drug delivery Part 2: Nanostructures for drug delivery. Trop. J. Pharm. Res. 2009;8(3):275-87.

Pabreja K, Dua K. Comparative evaluation of in situ intestinal absorption of aceclofenac from solid dispersi ons, $\beta$-cyclodextrin complexes and co-precipitates in rats. Bull. Pharm. Res. 2011;1(1):26-30.

Patel TB, Patel LD, Patel TR, Suhagia BN. Artificial neural as tool for quality by design in formulation development of solid dispersion of fenofibrate. Bull. Pharm. Res. 2015;5(1):20-7.

Pathak D, Dahiya S, Pathak K. Solid dispersion of meloxicam: Factorially designed dosage form for geriatric population. Acta Pharm. 2008;58(1):99-110. [DOI: 10.24 78/v10007-007-0048-y]

Prusty A. Formulation and in-vitro evaluation of amlodipine besylate-HP- $\beta$-CD inclusion complex incorporated mouth dissolving tablets. Bull. Pharm. Res. 2014;4(3):124-8.

Radhika P, Nagabhushanam MV, Venkata Ramana M. Preparation and characterization of Lornoxicam solid systems using cyclodextrins for improved bioavailability. Bull. Pharm. Res. 2015;5(3):101-7.

Riehemann K, Schneider SW, Luger TA, Godin B, Ferrari M, Fuchs H. Nanomedicine - Challenge and perspectives. Angew. Chem. Int. Ed. Engl. 2009;48(5):872-97. [DOI: 10.1002/anie.200802585].

Shegokar R, Muller RH. Nanocrystals: Industrially feasible multifunctional formulation technology for poorly soluble actives. Int. J. Pharm. 2010;399(1-2):129-39. [DOI: 10.101 6/j.ijpharm.2010.07.044]

Srivalli KMR, Mishra B. Drug nanocrystals: A way toward scale-up. Saudi Pharm. J. 2016;24(4):386-404. [DOI: 10.10 16/j.jsps.2014.04.007]

Teagarden DL, Baker DS. Practical aspects of lyophilisation using non-aqueous co-solvent systems. Eur. J. Pharm. Sci. 2002;15(2):115-33. [DOI: 10.1016/S0928-0987(01)0022 $1-4]$ 